\title{
Implications of Racial and Gender Differences In Patterns of Adolescent Risk Behavior for HIV And Other Sexually Transmitted Diseases
}

\begin{abstract}
CONTEXT: Sexual and substance use behaviors covary in adolescence. Prevalence of HIV and other sexually transmitted diseases (STDs) differs according to race and gender, yet few studies have systematically investigated risk behavior patterns by subgroup, particularly with nationally representative data.
\end{abstract}

\begin{abstract}
METHODS: A priori considerations and K-means cluster analysis were used to group 13,998 non-Hispanic black and white participants in the National Longitudinal Study of Adolescent Health, Wave 1, according to self-reported substance use and sexual behavior. Multinomial logit analyses examined racial and gender differences by cluster.
\end{abstract}

RESULTS: Among 16 clusters, the two defined by the lowest risk behaviors (sexual abstinence and little or no substance use) comprised $47 \%$ of adolescents; fewer than $1 \%$ in these groups reported ever having received an STD diagnosis.

The next largest cluster-characterized by sexual activity (on average, with one lifetime partner) and infrequent substance use-contained 15\% of participants but nearly one-third of adolescent with STDs. Blacks were more likely than whites to be in this group. Black males also were more likely than white males to be in three small clusters characterized by high-risk sexual behaviors (i.e., having had sex with a male or with at least 14 partners, or for drugs or money). Black females generally were the least likely to be in high-risk behavior clusters but the most likely to report STDs.

CONCLUSIONS: Adolescents' risk behavior patterns vary by race and gender, and do not necessarily correlate with their STD prevalence. Further investigation of adolescents' partners and sexual networks is needed.

Perspectives on Sexual and Reproductive Health, 2004, 36(6):239-247

HIV was the seventh-leading cause of death among U.S. 15-24-year-olds in 2002, and at least half of all newly infected persons in the country are younger than $25 .{ }^{1}$ This represents about 20,000 new HIV infections among youth annually. ${ }^{2}$ In addition, adolescents and young adults are at higher risk than other age-groups for sexually transmitted diseases (STDs) that can increase the risk of HIV infection. ${ }^{3}$ These troubling patterns exist even though youth are increasingly postponing sexual initiation and using condoms once they become sexually active. ${ }^{4}$

Females and non-Hispanic blacks are disproportionately affected by STDs. For example, in 2001, the gonorrhea infection rate among 15-19-year-old black females was almost twice as high as that for black males, and 18 times as high as the rate for white females; the reported rate was lowest among white males. For the same year, the rate of chlamydia among 15-19-year-old black females was 5-6 times as high as those for black males and white females of the same ages; again, the rate was lowest among white males. ${ }^{5}$ Among youth aged 13-24, 56\% of HIV diagnoses occur in blacks.

Racial disparities are large among disadvantaged youth. For example, among U.S. Job Corps entrants in the 1990s, the HIV infection rate among young black women was seven times as high as that among young white women. ${ }^{6}$ Such disparity is also particularly marked for young black men who have sex with men. In a large survey of young, urban men who have sex with men, the prevalence of HIV infection was
$14 \%$ among blacks, compared with 3\% among whites. ${ }^{7}$

In adolescents and young adults, HIV infections are mostly acquired through sexual activity, although drug use also puts young people at risk. ${ }^{8}$ Sharing needles is the direct drug-related route; however, persons with drug addiction may have sex with multiple partners to obtain drugs or money to buy drugs, thereby infecting otherwise low-risk individuals. Moreover, noninjection-drug use may reduce inhibitions, resulting in sexual risk-taking, although research on this has been mixed regarding adolescents. ${ }^{9}$

These risk behaviors tend to covary; in adolescent and young adult populations, substance use is positively related to sexual initiation, frequency and risk-taking. ${ }^{10}$ Covariation is important because it indicates that targeting single STD risk behaviors may be ineffective. Furthermore, demonstration of a single or predominant pattern of behavioral covariation would imply that different risk behaviors share causal antecedents; after these common antecedents are identified, targeting them in prevention and intervention efforts should reduce STD risk. However, relatively little research has systematically investigated whether a single pattern adequately captures risk behavior covariation, and whether that pattern is similar across genders and races. ${ }^{11}$ Identification of multiple patterns of risk behavior covariation would suggest potential differences in the risk of STDs, including HIV, among adolescents showing those patterns and the possibility that distinct developmental pathways culminate
ByCarolyn

Tucker Halpern,

Denise Hallfors,

Daniel J. Bauer,

Bonita Iritani,

Martha W. Waller

and Hyunsan Cho

Carolyn Tucker

Halpern is associate professor of maternal and child health, and Martha W. Waller is doctoral student, both at the School of Public Health, University of

North Carolina at

Chapel Hill. Denise

Hallfors is senior

research scientist, and

Bonita Iritani and

Hyunsan Cho are associate research scientists, all at the Pacific Institute for Research and Evaluation, Chapel Hill, NC. Daniel J.

Bauer is assistant professor of psychology, University of North Carolina at Chapel Hill. 


\begin{tabular}{|c|c|}
\hline Characteristic & $\begin{array}{l}\% \\
(\mathrm{~N}=13,998)\end{array}$ \\
\hline \multicolumn{2}{|l|}{ Gender } \\
\hline Female & 49.4 \\
\hline Male & 50.6 \\
\hline \multicolumn{2}{|l|}{ Race } \\
\hline White & 80.8 \\
\hline Black & 19.2 \\
\hline \multicolumn{2}{|l|}{ Parent's education } \\
\hline$<$ H.S. & 9.2 \\
\hline H.S. graduate/GED & 33.1 \\
\hline Some college & 22.5 \\
\hline zcollege graduate & 35.2 \\
\hline \multicolumn{2}{|l|}{ Family structure } \\
\hline Two parents & 70.5 \\
\hline Single mother & 20.7 \\
\hline Single father & 3.2 \\
\hline Other & 5.8 \\
\hline \multicolumn{2}{|c|}{ Current grade in school } \\
\hline $7-8$ & 34.1 \\
\hline $9-10$ & 32.8 \\
\hline $11-12$ & 31.3 \\
\hline Not in schoolt & 1.9 \\
\hline Total & 100.0 \\
\hline $\begin{array}{l}\text { †Students who appea } \\
\text { Wave } 1 \text { in-home inter } \\
\text { ous reasons (e.g., sus } \\
\text { Percentages are weig } \\
\text { grades } 7-12 \text {. }\end{array}$ & $\begin{array}{l}\text { re were eligible for th } \\
\text { not in school for vari } \\
\text { y, dropped out). Note } \\
\text { estimates for youth in }\end{array}$ \\
\hline
\end{tabular}

in different patterns of risk behavior. Identifying distinct patterns of risk and understanding the particular processes leading to those patterns are critical steps in developing and targeting risk prevention efforts.

Some research shows that the consistency and magnitude of covariation among risk behaviors related to sex and drug use vary across adolescent populations; in particular, associations between substance use and sexual risk-taking are less strong for black youth than for white youth. ${ }^{12}$ Given the overrepresentation of black youth among STD cases, an understanding is critically needed regarding differences between blacks and whites in the patterns of risk-taking that underlie these outcomes. However, studies have shown conflicting results regarding racial differences in risk behavior covariation; this is explained, in part, by a lack of nationally representative adolescent samples, and by differences in study design or statistical adjustment for important factors confounded with race, such as socioeconomic status. However, findings have been inconsistent even among samples with similar socioeconomic status. For example, Stanton and colleagues found that sexual activity typically did not covary with drug activities of low-income, urban black adolescents, whereas Farrell and colleagues found similar covariations for low-income blacks and whites. ${ }^{13}$

Adolescent risk behavior covariation also differs between genders. For example, at any adolescent age, engagement in risk behaviors, and in multiple risk behaviors, is more likely among males than among females. ${ }^{14}$ Findings also suggest that sexual risk behavior covariation with substance use is less likely among females than among males, again suggesting different dynamics of risk-taking. ${ }^{15}$

The first steps toward understanding the processes that lead to different risk behavior patterns and, ultimately, reducing STD risk are to identify the patterns of drug use and risky sexual activity that exist among youth, determine if these behavioral patterns and associated STD risk are similar for the groups who are the most and the least likely to be affected by STDs (blacks and non-Hispanic whites, respectively), and examine the association between behavioral patterns and STD risk. These steps can be facilitated by applying a person-centered analytic approach-that is, by examining, through cluster analysis, how people group together on the basis of the similarity of their risk behavior patterns. Similar to factor analysis, which groups variables together, cluster analysis groups individuals, on the assumption that risk behaviors often occur together and interact with each other. By combining individuals with similar behavior patterns, cluster analysis allows an examination of the interaction of all the behaviors, resulting in a parsimonious model and a holistic strategy for understanding youth behavior. ${ }^{16}$ Such person-oriented approaches may capture reliable patterns of problem behavior that reflect distinct causes with differing implications for later risk; by so doing, they may improve efforts to identify adolescents with a particularly high STD risk, and may increase the specificity and the sensitivity of prevention interventions.

Conceptually and empirically derived typologies have been used to examine variation in common adolescent problem behaviors. ${ }^{17}$ For example, Zweig and colleagues, using data on high school students participating in Wave 1 of the National Longitudinal Study of Adolescent Health (Add Health), generated four clusters, distinguished by patterns of eight diverse health risk behaviors examined. ${ }^{18}$ The authors concluded that multiple "problem behavior syndromes" exist, and that these syndromes differ for males and females. Although risk groups differed significantly by race and ethnicity, Zweig and colleagues noted that race and ethnicity, age and family income contributed little to prediction of risk group membership. However, the authors did not report how much of the variance in risk behavior the four-cluster solution accounted for; therefore, the adequacy of the cluster solution and the reliability of associations with it are unclear.

We used a similar analytic approach to identify adolescent groups sharing behavioral risk patterns that put them at risk for STDs other than HIV and, consequently, for HIV infection. We performed a detailed examination of risk behaviors specifically related to STDs to identify adolescents who are members of small but high-risk groups. The analysis consequently allows for a relatively large number of homogeneous clusters, or groups of adolescents; this is meant to capture behavioral risk patterns that increase adolescents' risk for STDs.

Our study addresses three questions. What patterns of sexual activity and drug-use behaviors exist in a nationally representative sample of black and white adolescents? 
Which demographic characteristics are shared by adolescents who show particular risk behavior patterns? In which adolescent clusters are STDs highly prevalent, and how does prevalence correspond to these clusters' demographic composition?

\section{METHODS \\ Add Health}

Data used in these analyses come from the contractual data set of Add Health, Wave 1. Add Health began with a school sample representing a stratified probability sample of all U.S. high schools. More than 90,000 adolescents in grades 7-12 from 132 schools completed questionnaires. All students who completed an in-school questionnaire or who were listed on a school roster (including those not attending school) were eligible for inclusion in an in-depth, inhome follow-up. A representative sample and several special samples selected on the basis of responses to the school questionnaire were selected for in-home interviews. ${ }^{19}$ Inhome interviews were completed during April-December 1995 with 20,745 adolescents; this represented 79\% of eligible respondents. A study comparing interviewed adolescents with dropouts found unbiased population estimates. ${ }^{20}$ In-home questionnaires were administered via laptop computer; audio computer-assisted self-interview was used to collect information on sensitive topics, such as sexual activity. All informed consent forms, questionnaires and procedures used in Add Health were reviewed and approved by the University of North Carolina at Chapel Hill Institutional Review Board for the Protection of Human Subjects, at the School of Public Health.

\section{Measures}

Gender is a self-reported measure. Age was calculated as the date of birth subtracted from the interview date, rounded to two decimal places. Race is based on self-reports: Respondents were asked to report all applicable races and to identify the one that best described them. By using information from multiple survey items, we constructed four racial groups: white, black, Asian or Pacific Islander, and Native American.

Parent's education represents the highest educational level achieved by respondents' resident father or mother figure, as reported by the adolescent. In accordance with common practice, this variable and information on family structure serve as socioeconomic status indicators. We chose highest parental education and family structure as socioeconomic status indicators because 29\% of black respondents and $20 \%$ of whites lacked income data.

Household roster information was used to construct a variable for family structure. (The categories were two parents, single mother, single father and other. $)^{21}$

Respondents were asked whether a doctor or nurse had ever told them that they had chlamydia, syphilis, gonorrhea, HIV or AIDS, genital herpes, genital warts, trichomoniasis or hepatitis B virus. Respondents reporting yes for any of these were coded one.
TABLE 2. Percentage distribution of participants, by cluster, and behavioral patterns defining each cluster

Cluster type and behavioral patterns

Light substance dabblers-infrequent or no current use of substances $†$

None have had sex

Abstainers—none have ever used substancest or had sex

Sex dabblers-all have had sex

Median no. of partners $=1$

$60 \%$ used a condom at last sex

Infrequent use of substancest

Drinkers-all consumed alcohol in past 12 mos.

$49 \%$ report binge drinking

Infrequent or no illicit drug use

None have had sex

Smokers-all smoke cigarettes daily

Infrequent use of alcohol/illicit drugs

$62 \%$ have had sex

Alcohol-and-sex dabblers_-all drink occasionally; all have had sex

Infrequent tobacco/illicit drug use

Binge drinkers-all binge frequently

Infrequent cigarette, marijuana and other drug use

$60 \%$ binge $\geq 1$ time/wk.

$45 \%$ have had sex

Heavy dabblers-all smoke, drink and binge drink with moderate frequency

$45 \%$ use marijuana; few use other illicit drugs

$91 \%$ have had sex

Combination sex and drug use-all have had sex; all used alcohol/illicit drug at last sex

Marijuana users-all use marijuana frequently; few have used other illicit drugs

$94 \%$ use alcohol

$79 \%$ smoke cigarettes

$74 \%$ have had sex

Multiple partners-all report $\geq 14$ sexual partners

$75 \%$ report low or moderate use of substances $\dagger$

Sex for drugs or money-all have had sex for drugs or money

$50 \%$ report low or moderate use of substancest

Median no. of partners $=3$

High marijuana use and sex —all use marijuana frequently; all have had sex All used alcohol/other drug at last sex

$82 \%$ have had $>1$ partner (median $=6$ )

Marijuana and other drug users-95\% report heavy marijuana use; all use other illicit drugs $68 \%$ have had sex

$28 \%$ used alcohol/other drug at last sex

Injection-drug users-all have injected drugs

$82 \%$ have had sex

Median no. of partners $=4$

Males who have sex with males - all are males who have had sex with another male $78 \%$ have had multiple partners (median $=5$ )

$40 \%$ used marijuana in past 30 days

$50 \%$ use alcohol $\geq 1$ time $/ \mathrm{mo}$.

$17 \%$ have had sex for drugs or money

Total

100.0

†Alcohol, tobacco and other drugs. Note: Percentages are weighted to yield national probability estimates for youth in grades $7-12$.

\section{Construction of Clusters}

Each participant was assigned to one of 16 clusters on the basis of his or her patterns of substance use and sexual behavior. Respondents were asked about ever-use of injection drugs, frequency of binge drinking and frequency of use of other substances-cigarettes, alcohol, marijuana and 


\begin{tabular}{|c|c|c|}
\hline Cluster & $\begin{array}{l}\% \text { in each cluster with } \\
\text { an STD diagnosis }\end{array}$ & $\begin{array}{l}\% \text { distribution } \\
\text { of adolescents with } \\
\text { an STD diagnosist }\end{array}$ \\
\hline Light substance dabblers & $0.2(0.0-0.5)$ & $1.5(0.4-4.8)$ \\
\hline Abstainers & 0.0 & 0.0 \\
\hline Sex dabblers & $4.9(3.8-6.4)$ & $29.1(23.2-35.7)$ \\
\hline Drinkers & $0.1(0.0-0.5)$ & $0.2(0.0-1.4)$ \\
\hline Smokers & $2.3(1.4-3.8)$ & $6.9(4.4-10.6)$ \\
\hline Alcohol-and-sex dabblers & $6.2(4.2-8.9)$ & $13.6(9.7-18.7)$ \\
\hline Binge drinkers & $2.0(1.0-3.7)$ & $3.6(1.9-6.7)$ \\
\hline Combination sex and drug use & $5.1(3.0-8.7)$ & $7.0(4.0-12.2)$ \\
\hline Heavy dabblers & $4.5(2.8-7.1)$ & $6.6(4.1-10.6)$ \\
\hline Marijuana users & $6.1(3.4-10.4)$ & $4.2(2.3-7.3)$ \\
\hline Multiple partners & $18.1(11.6-27.2)$ & $9.8(6.2-15.4)$ \\
\hline Sex for drugs or money & $15.9(9.9-24.7)$ & $7.5(4.9-11.4)$ \\
\hline High marijuana use and sex & $8.3(3.6-17.8)$ & $3.9(1.8-8.0)$ \\
\hline Marijuana and other drug users & $1.1(0.2-6.4)$ & $0.3(0.0-1.5)$ \\
\hline Injection-drug users & $19.7(10.9-33.1)$ & $5.0(2.7-9.2)$ \\
\hline Males who have sex with males & $7.8(2.6-21.6)$ & $1.0(0.3-2.8)$ \\
\hline
\end{tabular}

$+\mathrm{N}=367$. Notes: Values in parentheses are $95 \%$ confidence intervals. Percentages are weighted to yield national probability estimates for youth in grades 7-12.

other drugs. Responses regarding ever having used substances were used to define abstainers. Questions on sexual behavior asked about ever having had vaginal intercourse, having used a condom at most recent intercourse, having used alcohol or another drug at most recent intercourse, ever having had sex for drugs or money, and having had sexual intercourse with another male in the past 18 months (asked of males only).

Two general approaches can be used to classify individuals into groups. One is a top-down, or theory-driven, approach, whereby groups are formed a priori through specific decision rules (e.g., children of alcoholics or clinically depressed patients). The other is a bottom-up, or datadriven, approach, in which groups are defined inductively on the basis of the modal patterns in the data. Typically, the former approach is preferred, because of its greater reliance on theory. However, the latter approach is especially valuable when a clear theoretical basis for grouping individuals does not exist. Coupled with appropriate repli-

*The alternative strategy was to cluster the total sample; however, because cluster analysis allows some within-group heterogeneity, clustering the total sample would probably result in the combining of adolescents who had never engaged in sex or drug use and those who had. Because we believe these two groups have important theoretical distinctions, we separated the abstainers a priori.

Other investigators have constructed an abstainers cluster before inductive analysis (source: Rouillier P et al., Drinking patterns in French adult mena cluster analysis of alcoholic beverages and relationship with lifestyle, European Journal of Nutrition, 2004, 43(2):69-76). In addition, we anticipated that because the most extreme risk groups would likely be small, they probably would merge into heterogeneous clusters defined by wide-ranging risk behaviors. In this scenario, cluster members with extreme risk behaviors could distort the analysis by pulling the mean of their cluster away from moderate risk levels. Empirically forcing such outliers into otherwise homogeneous clusters is not recommended (source: Bergman LR and Magnusson $\mathrm{D}$, A person-oriented approach in research on developmental psychopathology, Development and Psychopathology, 1997, 9(2):291-319).

TSAS PROC FASTCLUS has a nearest-centroid sorting algorithm that accounts for probability weights and missing data. Given the chosen number of clusters, the clustering algorithm determines the cluster structure that minimizes the summed within-cluster variance of the variables, equivalently maximizing the between-cluster centroid differences. (Additional details about the analysis are available from the authors.) cation and validation efforts, a data-driven approach allows for the identification of "natural structures" of individual differences that might otherwise be relegated to error variance when hypotheses are insufficiently specific. ${ }^{22}$ We combined the two approaches, using specific decision rules to form groups of particular theoretical interest. Beyond these groups, we had no theoretical basis for deciding the most salient patterns in the data; therefore, we used K-means cluster analysis to inductively define and group the remaining individuals according to their empirical similarity on HIV or other STD risk behaviors.

In creating clusters of adolescents, we limited the initial sample to respondents with valid grand sample weights; this reduced the sample to 18,924 adolescents. We standardized all variables to place them on the same scale, a practice recommended for cluster analysis. ${ }^{23}$

- Clusters defined a priori. We defined four clusters a priori. Three of these reflect uncommon behaviors in this age-group that put individuals at greatest risk for HIV. Because K-means cluster analysis becomes unreliable with extreme observations, uncommon risk behaviors were examined first. These three clusters are injection-drug users, males who have sex with males, and adolescents who have had sex for drugs or money. The cluster of injection-drug users was constructed first; it comprises all respondents reporting ever-use of injected drugs. Next, males were assigned to the cluster of males who have sex with males if they reported having had sexual intercourse with another male in the past 18 months and never having used injection drugs. The sex for drugs or money cluster comprises anyone who reported ever having had sex for drugs or money, but never having used injection drugs, and who was not a male who has sex with males. The fourth a priori cluster was abstainers, defined as individuals who reported never having had sexual intercourse and never having used cigarettes, alcohol or illicit drugs.

- Inductively derived clusters. The remaining clusters were derived from a K-means cluster analysis, performed by using the PROC FASTCLUS procedure in SAS, to identify the modal risk patterns of participants not assigned to one of the predefined clusters. ${ }^{* 24}$ This procedure forces clusters to be as distinct as possible. ${ }^{\dagger}$ To choose the optimal number of clusters for respondents unassigned to an a priori cluster, we compared models involving 4-16 clusters. Preliminary, parallel cluster analyses were performed for participants in each racial, gender and school grade combination; these analyses yielded the same basic cluster structure for each subsample. (That is, the same risk patterns appeared in each analysis; however, the proportion of individuals in specific clusters often varied according to race, gender and grade level.) Given this structural similarity, the cluster analysis was repeated with the total sample to facilitate comparisons in cluster membership by race and gender. Taking into account high variance accounted for in risk behaviors (i.e., high $\mathrm{R}^{2}$ ), parsimony, interpretability and the retention of clusters identified in preliminary analyses as being composed primarily of one race, gender or grade level, we selected a 12-cluster solution from 
the K-means analysis. The 16 clusters account for $76 \%$ of the variance in the adolescents' risk-taking behavior patterns. The multiple parallel analyses conducted by race, grade level and gender showed substantially similar cluster solutions, confirming the replicability of overall patterns.

\section{Multinomial Logit Analyses}

We examined racial and gender differences in cluster membership by performing two multinomial logistic regression analyses. Our analyses assessed only non-Hispanic whites and non-Hispanic blacks because these groups are, respectively, the least and most affected by HIV and other STDs, and our research questions focus on racial and gender differences. After exclusions, our sample comprised 13,998 participants.

In the multinomial analyses, cluster membership was the dependent variable; age, parent's education and family structure were control variables, and race and gender were key predictors. Because the males who have sex with males cluster, by definition, included only male participants, this cluster was excluded from a multinomial logit performed for both male and female participants. This cluster was then evaluated in a parallel analysis involving males only. Abstainers were the reference group in both models. To determine whether behavioral risk patterns (i.e., cluster membership) varied by both race and gender, we tested interactions between race and gender in cluster membership.

The overall sample was evenly split between females and males; 19\% of participants were black (Table 1, page 240). More than half of participants had a parent with at least some college education. The majority of participants lived in two-parent families. The median age of the sample was 16 (range, 12-22).

Sampling weights were applied in all analyses, and the clustering of adolescents within schools was taken into account in the calculation of variance estimates by using the robust option (Huber-White correction) in Stata software. ${ }^{25}$ Missing data in the multinomial models were handled by using listwise deletion. All reported percentages are weighted.

\section{RESULTS}

\section{Behavioral Risk Patterns That Define Clusters}

The cluster labels (Table 2, page 241) reflect the one or two dominant behaviors in each group. The clusters reflect different patterns of drug use and sexual activity, and variations in the frequency of engaging in risk behaviors or the proportions of respondents reporting those behaviors. Smaller clusters are typically defined by extremely high risk behaviors that few adolescents report. For example, the largest cluster (containing $24 \%$ of respondents) is light substance dabblers, who are characterized by infrequent or no current use of alcohol, tobacco or other drugs and no sexual experience. Abstainers, the next largest cluster (23\%), have never used alcohol, tobacco or other drugs and have never had sex. The third largest cluster, sex dabblers (15\% of the sample), are characterized by sexual experience with relatively few lifetime partners (75\% reported $1-2$ partners) and low
TABLE 4. Percentage distribution of participants, by cluster, according to race and gender

\begin{tabular}{|c|c|c|c|c|}
\hline \multirow[t]{2}{*}{ Cluster } & \multicolumn{2}{|l|}{ Black } & \multicolumn{2}{|l|}{ White } \\
\hline & $\begin{array}{l}\text { Female } \\
(\mathrm{N}= \\
2,139)\end{array}$ & $\begin{array}{l}\text { Male } \\
(\mathrm{N}= \\
1,891)\end{array}$ & $\begin{array}{l}\text { Female } \\
(\mathrm{N}= \\
5,079)\end{array}$ & $\begin{array}{l}\text { Male } \\
(\mathrm{N}= \\
4,889)\end{array}$ \\
\hline Light substance dabblers & 21.6 & 17.2 & 24.7 & 26.6 \\
\hline Abstainers & 24.3 & 16.5 & 24.6 & 22.0 \\
\hline Sex dabblers & 31.9 & 35.4 & 10.9 & 8.9 \\
\hline Drinkers & 3.5 & 1.2 & 8.6 & 8.8 \\
\hline Smokers & 1.0 & 1.4 & 10.1 & 7.4 \\
\hline \multicolumn{5}{|l|}{ Alcohol-and-sex } \\
\hline dabblers & 8.0 & 6.3 & 5.7 & 4.3 \\
\hline Binge drinkers & 3.2 & 4.1 & 3.1 & 6.1 \\
\hline \multicolumn{5}{|l|}{ Combination sex } \\
\hline and drug use & 2.8 & 2.6 & 3.5 & 3.6 \\
\hline Heavy dabblers & 0.3 & 1.0 & 4.2 & 4.3 \\
\hline Marijuana users & 0.8 & 1.3 & 1.4 & 2.3 \\
\hline Multiple partners & 0.9 & 6.0 & 0.7 & 0.9 \\
\hline Sex for drugs or money & 0.6 & 2.7 & 0.6 & 1.4 \\
\hline High marijuana use and sex & 0.4 & 2.5 & 0.9 & 1.2 \\
\hline \multicolumn{5}{|l|}{ Marijuana and other } \\
\hline drug users & 0.2 & 0.1 & 0.6 & 0.7 \\
\hline Injection-drug users & 0.2 & 0.4 & 0.4 & 1.0 \\
\hline \multicolumn{5}{|l|}{ Males who have sex } \\
\hline with males & 0.0 & 1.4 & 0.0 & 0.5 \\
\hline Total & 100.0 & 100.0 & 100.0 & 100.0 \\
\hline
\end{tabular}

Note: Percentages are weighted to yield national probabilty estimates for youth in grades 7-12.

levels of substance use. Patterns that reflect greater substance use, or the combination of increased substance use and sexual activity, are less common. The smallest cluster (comprising fewer than $1 \%$ of participants) is males who have sex with males; most members of this cluster report risky sexual behavior and use of alcohol, tobacco or other drugs.

\section{Bivariate Findings}

Six percent of sexually experienced respondents reported receiving an STD diagnosis. Black females were the most likely to report a diagnosis (18\%), whereas white males were least likely (fewer than 2\%). Diagnoses were reported by $6 \%$ of black males and 6\% of white females. More than half (59\%) of adolescents with an STD history reported receiving a diagnosis of chlamydia; of these respondents, $70 \%$ were female.

In each of three clusters-adolescents who had had sex for money or drugs, adolescents who had had multiple partners and injection-drug users-almost one in five respondents reported a previous STD diagnosis (Table 3, page 242). These clusters' contribution to the total group of participants with a previous STD-22\%-seems disproportionately high, given that each one comprises no more than approximately $1 \%$ of the sample. Two clusters with less-extreme risk profiles, sex dabblers and alcohol-and-sex dabblers, also contribute disproportionately to the group of adolescents who reported an STD. These two clusters represent about 20\% of the sample but account for $43 \%$ of youth reporting an STD. Both of these clusters are defined primarily by sexual activity (all members are sexually experienced), although members of the latter cluster reported more risky behavior.

The clusters with the highest proportions of white females and males were abstainers, light substance dabblers, 
TABLE 5. Relative risk ratios (and 95\% confidence intervals) showing black participants' likelihood of belonging to a cluster, compared with whites', by gender

\begin{tabular}{lll} 
Cluster & Females & Males \\
\hline Multiple partners & $1.0(0.3-3.0)$ & $7.9(4.5-13.9)^{* * *}$ \\
Sex dabblers & $2.3(1.7-3.0)^{* * *}$ & $4.9(3.6-6.9)^{* * *}$ \\
Sex for drugs or money & $0.6(0.2-1.6)$ & $1.9(1.0-3.7)^{*}$ \\
High marijuana use and sex & $0.2(0.1-0.8)^{*}$ & $1.9(0.9-3.9)$ \\
Heavy dabblers & $0.1(0.0-0.1)^{* * *}$ & $0.3(0.1-0.6)^{* *}$ \\
\hline *p $\leq$.05. **p $\leq .01 . * *$. $\leq .001$. Notes: Abstainers is the reference group. Data are \\
adjusted for age, parent's education level and family structure.
\end{tabular}

smokers, drinkers and sex dabblers (Table 4, page 243). Similarly, the clusters with the highest proportions of black females and males are the abstainers, light substance dabblers and sex dabblers clusters; however, the proportions of black youth in the sex dabblers cluster are much higher than the proportions of white youth appearing in this cluster. In addition, the alcohol-and-sex dabblers cluster contains higher proportions of black male and female participants than white male and female participants. These demographic patterns of cluster membership parallel patterns of race and cluster differences in past STDs.

As was seen in Table 3, the sex dabblers cluster and the alcohol-and-sex dabblers cluster are notable because of their disproportionate contribution to reports of diagnosed STDs. Black youth are more likely than white youth to appear in these clusters, especially the sex dabblers cluster-a pattern consistent with greater proportions of black youths' reporting a past STD diagnosis. Thus, the clusters that contribute disproportionately to reports of past STDs are populated disproportionately by youth who are more likely to report an STD.

\section{Multivariate Findings}

Older adolescents are more likely than younger adolescents to be in any cluster other than the abstainers cluster (not shown). Adolescents who live with two parents are more likely than other adolescents to be abstainers; moreover, participants who have better educated parents are more likely than those with less educated parents to be abstainers.*

Cluster membership varies by both race and gender (that is, we found a significant interaction between race and gender) for five clusters. Inclusion of the interaction term improved the fit of the model, according to the Wald test (chi-square $=70.66, \mathrm{p}<.001$ ) and the likelihood ratio test (chi-square $=99.94, \mathrm{p}<.001$ ). These five clusters-multiple partners, sex dabblers, sex for drugs or money, high mari-

\begin{tabular}{|c|c|}
\hline Cluster & Relative risk ratio \\
\hline Injection-drug users & $3.2(1.8-5.7)^{* * *}$ \\
\hline Binge drinkers & $2.1(1.6-2.7)^{* * *}$ \\
\hline Marijuana users & $1.8(1.3-2.6)^{* *}$ \\
\hline Light substance dabblers & $1.2(1.0-1.4)^{*}$ \\
\hline
\end{tabular}

juana use and sex, and heavy dabblers-are defined primarily by sexual risk-taking. In analyses assessing the relative risk of black males' (compared with whites') being in each cluster, conditional on gender and with adjustment for age and socioeconomic variables, racial comparisons are significant for each cluster except one (Table 5). For females, three of five clusters show significant racial differences. Black females are more likely than their white counterparts to appear in the sex dabblers cluster. In contrast, black females are considerably less likely than white females to be in the heavy dabblers cluster and the high marijuana use and sex cluster.

Compared with white males, black males are almost eight times as likely to be in the multiple partner cluster rather than in the abstainer cluster. In addition, black males are almost five times as likely to be in the sex dabblers cluster, and almost twice as likely to appear in the sex for drugs or money cluster. However, black males are only about onethird as likely as whites to be in the heavy dabblers cluster.

The remaining 10 clusters show no race-gender interactions; however, significant differences (main effects) between males and females appear for four clusters, each of them defined primarily by substance use: injection-drug users, binge drinkers, marijuana users and light substance dabblers. Males are more likely than females to appear in these clusters rather than in the abstainer cluster (Table 6). The relative risk for membership in the injection-drug users cluster is the largest; males are more than three times as likely as females to be in this cluster.

Race has main effects in nine clusters (Table 7). Three of these clusters (injection-drug users, marijuana users and light substance dabblers) also differ by gender. In general, blacks are more likely than whites to appear in clusters defined primarily by sexual activity (males who have sex with males, alcohol-and-sex dabblers). However, blacks are significantly less likely than whites to appear in clusters defined primarily by substance use (smokers, marijuana and other drug users, drinkers, injection-drug users, marijuana users, combination sex and drug users, and light substance dabblers).

\section{DISCUSSION}

We used a holistic, person-centered approach to sort adolescents into 16 groups, or clusters, that capture almost $80 \%$ of the variance in their substance use and sexual risk-taking behavior patterns. The clusters reflect distinct patterns of drug use and sexual activity, and gradations in the extent of those activities. Our findings therefore support, in a large, nationally representative sample, what earlier literature has documented-that drug use and sexual be-

*To examine whether the absence of income in our models obscures important socioeconomic differences with implications for racial patterns of cluster membership, we conducted parallel analyses with and without use of a family income predictor. Including income did not eliminate significant racial differences by cluster, except for these groups: marijuana users, males who have sex with males and injection-drug users. Whether these few differences are explained by the additional adjustment for income, or by a selection bias due to nonrandom missing data, is unclear. 
TABLE 7. Relative risk ratios (and 95\% confidence intervals) showing black participants' likelihood of belonging to a cluster, compared with whites'

Cluster

Relative risk ratio

Males who have sex with males

Alcohol-and-sex dabblers

Light substance dabblers

Smokers

Marijuana and other drug users

Drinkers

Injection-drug users

Marijuana users

Combination sex and drug use

${ }^{*} p \leq .05$. ${ }^{* * *} p \leq .001$. Notes: Abstainers is the reference group. Data are adjusted

for age, parent's education level and family structure.

haviors covary among adolescents. However, our analyses further demonstrate that there are multiple, distinctive patterns of risk behavior covariation, and that the assumption of a single "problem behavior syndrome" is incorrect. The existence of coherent, distinct behavioral risk patterns suggests there likely are different causal pathways underlying the patterns, pathways that must be recognized and understood before appropriate prevention efforts can be developed. Clues to these causal pathways can probably be identified in research that simultaneously considers behavioral patterns and the social networks in which they are embedded.

Although the same cluster structure is evident for black and white males and females, some behavioral patterns are more characteristic of one demographic group than others. After adjustment for age and socioeconomic indicators, black adolescents are less likely than white adolescents to appear in risk clusters defined primarily by substance use (e.g., drinkers) or clusters defined by high substance use and sexual risk-taking (e.g., smokers, heavy dabblers). In contrast, sexual activity does not necessarily covary with substance use among black adolescents, who are more likely than white adolescents to appear in clusters defined primarily by sexual risk-taking. For example, one-third of black adolescents appear in the sex dabblers cluster, which is characterized by very low drug use, compared with only about $10 \%$ of white adolescents. Some racial differences vary by gender (e.g., membership in the sex dabblers and the multiple partners clusters), with males tending to show racial differences of greater magnitude than females.

Gender has significant main effects on cluster membership: Males are more likely than females to appear in clusters defined primarily by substance use (light substance dabblers, binge drinkers, marijuana users and injection-drug users) but also (except for light substance dabblers) high levels of sexual activity. These patterns of gender differences support earlier findings that females, compared with males, are less likely to show covariation of high drug use and sexual risk-taking. ${ }^{26}$ The reliability and generalizability of earlier findings regarding gender and, particularly, racial differences in risk behavior patterns are unclear because of sample and analytic limitations. ${ }^{27}$

Consistent with earlier work on more generalized risk be- havior, the largest clusters in our sample of adolescents are abstainers and light substance dabblers. ${ }^{28}$ As would be expected, only a small proportion of these adolescents reported a past STD diagnosis. Smaller clusters, characterized by risky sexual activity or relatively high drug use, have higher proportions of members who reported a past STD diagnosis. Clusters defined by sexual risk-taking are more likely to have members who reported an STD, and after adjustment for socioeconomic status, they are more likely to include black adolescents, who are overrepresented in the STD-infected population. Two particularly high risk sexual patterns-those characterizing the multiple partners cluster and the males who have sex with males cluster-are especially characteristic of black males, whose risk of STDs is higher than that of white males. These findings underscore the importance of identifying behavioral patterns that vary across demographic groups; however, because the STD data are selfreported, the associations may also reflect a higher likelihood of getting tested among individuals in these clusters.

Our results support the important implications of behavioral variation for acquiring STDs; they also indicate that the relationship between STDs and behavioral risk patterns must be placed in a broader context. This is indicated by our finding that sex dabblers, who are characterized by seemingly low-risk behavior (e.g., most have had one or two partners and use condoms), represent 15\% of the sample but account for almost one-third of past STD diagnoses. This cluster is dominated by black males and females. These youth, especially females, carry the greatest STD burden. The fact that members of this apparently low-risk cluster are more likely to report past STDs highlights that although an individual's pattern of sexual behavior and drug use is a significant predictor of STD risk, other factors are also important. ${ }^{29}$ The relatively low levels of sexual risk-taking among black females in the face of high STD prevalence indicate the need for information about the sexual patterns of their partners. Our findings suggest that adolescent males who exhibit high-risk sexual behavior patterns are crossing over to lower-risk adolescent groups for sexual partners, a pattern that has been documented among adults. ${ }^{30}$ Black females are likely to limit their sexual contacts to black males and to have partners who are two or more years their senior. ${ }^{31}$ The fact that black males are overrepresented in almost all of the groups at high risk of STDs identified here suggests that crossover between high-and low-risk groups may disproportionately elevate the risk for black females.

One in five sexually experienced black females in this nationally representative sample reported that they had received an STD diagnosis, compared with fewer than one in 10 white females. There is a tremendous need to understand the factors and processes that account for these differences. Racial and gender differences in risk behavior patterns have important implications for STD risk and, therefore, for our understanding of processes culminating in different patterns of risk behavior. However, crossover partnering from high- to low-risk groups, a behavior not

\section{The relatively}

low levels of sex-

\section{ual risk-taking}

among black

females in the

face of high STD

prevalence indi-

cate the need for

information

about the sexual

patterns of their

partners. 
captured in our analyses, likely accounts for part of the STD disparity. Yet recent efforts to examine how pressures regarding the selection of partners and other contextual factors may influence crossover partnering-accounting for some of the disparity between black and white femaleshave had only minimal explanatory power. ${ }^{32}$ Efforts to examine longitudinal STD patterns among adolescents have also identified few predictive factors beyond previous infection. ${ }^{33}$ These studies point to the need for research on adolescent populations that includes youth who are at seemingly diverse levels of risk, tracks patterns of sexual behavior over time through the lens of the whole individual, and documents the characteristics and sexual histories of partners. Including these elements could clarify the dynamics of adolescent partnering and sexual decision-making that are embedded in different sociocultural contexts.

Our analyses are limited by reliance on self-reports of STD diagnosis, because STD diagnoses may be underreported among adolescents. ${ }^{34}$ The more general issue of underestimation is likely more problematic for males, who may have fewer occasions than females for medical examinations and, therefore, for diagnosis. However, the associations demonstrated here between behavioral risk-taking patterns reflected in cluster membership and STD diagnosis are logical and support the utility of both the self-reported STD measure and the cluster groupings.

\section{REFERENCES}

1. Kochanek KD and Smith BL, Deaths: preliminary data for 2002, National Vital Statistics Reports, 2004, Vol. 52, No. 13; and Rosenberg PS and Biggar RJ, Trends in HIV incidence among young adults in the United States, Journal of the American Medical Association, 1998, 279(23): 1894-1899.

2. Weinstock $\mathrm{H}$ et al., Sexually transmitted diseases among American youth: incidence and prevalence estimates, Perspectives on Sexual and Reproductive Health, 2004, 36(1):6-10

3. Anderson RM, Transmission dynamics of sexually transmitted infections, in: Holmes KK et al., eds., Sexually Transmitted Diseases, third ed., New York: McGraw-Hill, 1999.

4. Centers for Disease Control and Prevention (CDC), Trends in sexual risk behaviors among high school students-United States, 1991-2001, Morbidity and Mortality Weekly Report, 2002, 51(38):856859; and Murphy JJ and Boggess S, Increased condom use among teenage males, 1988-1995: the role of attitudes, Family Planning Perspectives, 1998, 30(6):276-280 \& 303.

5. CDC, Sexually Transmitted Disease Surveillance, 2001, Atlanta: CDC, 2002; and Miller WC et al., Prevalence of chlamydial and gonococcal infections among young adults in the United States, Journal of the American Medical Association, 2004, 291(18):2229-2236.

6. CDC, Young people at risk: HIV/AIDS among America's youth, 2002 <http://www.cdc.gov/hiv/pubs/facts/youth.htm>, accessed Mar. 5, 2003; and Valleroy LA et al., HIV infection in disadvantaged out-of-school youth: prevalence for U.S. Job Corps entrants, 1990 through 1996, Journal of Acquired Immune Deficiency Syndromes and Human Retrovirology, 1998, 19(1):67-73

7. Valleroy LA et al., HIV prevalence and associated risks in young men who have sex with men, Journal of the American Medical Association, 2000, 284(2): 198-204

8. CDC, HIV/AIDS Surveillance Report: Cases of HIV Infection and AIDS in the United States, 2003, Vol. 15, p. 10, <http://www.cdc.gov/hiv/stats/ 2003surveillancereport.pdf>, accessed Nov. 16, 2004.

9. Cooper ML et al., Substance use and sexual risk taking among black adolescents and white adolescents, Health Psychology, 1994, 13(3):251262; and Fortenberry JD, Alcohol, drugs and STD/HIV risk among adolescents, AIDS Patient Care and STDs, 1998, 12(10):783-786.

10. Duncan SC et al., Exploring associations in developmental trends in adolescent substance use and risky sexual behavior in a high-risk population, Journal of Behavioral Medicine, 1999, 22(1):21-34; and Graves KL and Leigh BC, The relationship of substance use to sexual activity among young adults in the United States, Family Planning Perspectives, 1995, 27(1):18-22 \& 33.

11. Fortenberry JD, Adolescent substance use and sexually transmitted diseases risk: a review, Journal of Adolescent Health, 1995, 16(4):304308.

12. Ibid.; and Stanton B et al., Early initiation of sex and its lack of association with risk behaviors among adolescent African Americans, Pediatrics, 1993, 92(1):13-19.

13. Stanton B et al., 1993, op cit. (see reference 12); Farrell AD et al., Relationship between drug use and other problem behaviors in urban adolescents, Journal of Consulting Clinical Psychology, 1992, 60(5):705712 .

14. Lindberg LD et al., Multiple threats: the co-occurrence of teen health risk-behaviors, in: Department of Health and Human Services (DHHS), Trends in the Well-Being of America's Children and Youth, 1999, Washington, DC: DHHS, 2000.

15. Basen-Engquist K et al., Structure of health risk behavior among high school students, Journal of Consulting Clinical Psychology, 1996, 64(4):764-775.

16. Magnusson D, The logic and implications of a person-oriented approach, in: Cairns RB and Bergman LR, eds., Methods and Models for Studying the Individual, Thousand Oaks, CA: Sage Publications, 1998; and Bauer DJ, Person-centered approaches to data analysis, paper presented at the Merrill Center Conference: Modeling Developmental Processes in Ecological Context, Tempe, AZ, Mar. 4-6, 2004.

17. Windle M, An alcohol involvement typology for adolescents: convergent validity and longitudinal stability, Journal of Studies on Alcohol, 1996, 57(6):627-637; and Miller KS et al., Adolescent heterosexual experience: a new typology, Journal of Adolescent Health, 1997, 20(3):179186.

18. Zweig JM et al., Adolescent health risk profiles: the co-occurrence of health risks among males and females, Journal of Youth and Adolescence, 2001, 30(6):707-728; and Zweig JM et al., Predicting adolescent profiles of risk: looking beyond demographics, Journal of Adolescent Health, 2002, 31(4):343-353

19. Harris KM et al., The National Longitudinal Study of Adolescent Health: research design, 2003, <www.cpc.unc.edu/projects/addhealth/ design>, accessed Nov. 16, 2004

20. Udry J and Chantala K, Missing school dropouts in surveys does not bias risk estimates, Social Science Research, 2003, 32(2):294-311.

21. Harris KM, The health status and risk behavior of adolescents in immigrant families, in: Hernandez DJ, ed., Children of Immigrants: Health, Adjustment and Public Assistance, Washington, DC: National Academy Press, 1999

22. Bauer DJ et al., Alternative approaches to analyzing individual differences in the rate of early vocabulary development, Applied Psycholinguistics, 2002, 23(3):313-315.

23. Lorr M, Cluster Analysis for Social Scientists, San Francisco: JosseyBass, 1983.

24. SAS Institute, SAS/STAT User's Guide, Volume 2, version 6, fourth ed., Cary, NC: SAS Institute, 1990

25. Stata Corp., Stata Statistical Software: Release 7.0, College Station, TX: Stata Corp., 2001

26. Basen-Engquist K et al., 1996, op. cit. (see reference 15)

27. Ibid.; and Stanton B et al., 1993, op. cit. (see reference 12)

28. Zweig JM et al., 2001, op. cit. (see reference 18); and Zweig JM et al., 2002, op. cit. (see reference 18).

29. Ellen JM et al., Socioeconomic difference in sexually transmitted 
disease rates among black and white adolescents, San Francisco, 1990 to 1992, American Journal of Public Health, 1995, 85(11):1546-1548; and Santelli JS et al., The association of sexual behaviors with socioeconomic status, family structure and race/ethnicity among U.S. adolescents, American Journal of Public Health, 2000, 90(10):1582-1588.

30. Anderson RM, 1999, op. cit. (see reference 3).

31. Ford Ket al., American adolescents: sexual mixing patterns, bridge partners and concurrency, Sexually Transmitted Diseases, 2002, 29(1):1319.

32. Harawa NT et al., Do differences in relationship and partner attributes explain disparities in sexually transmitted disease among young white and black women? Journal of Adolescent Health, 2003, 32(3):187-191.

33. Crosby R, Leichliter JS and Brackbill R, Longitudinal prediction of sexually transmitted diseases among adolescents: results from a national survey, American Journal of Preventive Medicine, 2000, 18(4):312317.

34. Harrington KF et al., Validity of self-reported sexually transmitted diseases among African American female adolescents participating in an HIV/STD prevention intervention trial, Sexually Transmitted Diseases, 2001, 28(8):468-471.

\section{Acknowledgments}

This work was supported by the National Institute on Drug Abuse (grant R01-DA14496-01A1). The authors thank Carol A. Ford, for helpful feedback on an earlier draft of the manuscript; Guang Guo, for statistical consultation; Jon Hussey, for consultation on analyses and manuscript drafts; and Chris Wiesen, for SAS programming consultation and assistance. They also thank Jan Hendrickson-Smith and J. Richard Udry, for development of a comprehensive variable for race, and Kathleen Mullan Harris and Suzanne Ryan, for development of a measure of family structure.

This research uses data from Add Health, a program project designed by J. Richard Udry, Peter S. Bearman and Kathleen Mullan Harris, and funded by grant P01-HD31921 from the National Institute of Child Health and Human Development, with cooperative funding from 17 other agencies. Special acknowledgment is due Ronald R. Rindfuss and Barbara Entwisle for assistance in the original design. Persons interested in obtaining data files from Add Health should contact Add Health, Carolina Population Center, 123 W. Franklin Street, Chapel Hill, NC 27516-2524 (<www.cpc. unc.edu/addhealth/contract.html>).

Author contact: carolyn_halpern@unc.edu 